\title{
Research on Intelligent Temperature Control System of Automobile Heating Pad Based on Expert Control
}

\author{
Mingyue $\mathrm{Li}^{1,}$ a and Jian Fang ${ }^{2, \mathrm{~b}}$ \\ ${ }^{1}$ School of automotive Engineering, Jilin Engineering Normal University, Changchun, China \\ ${ }^{2}$ School of automotive Engineering, Jilin Engineering Normal University, Changchun, China \\ a254295092@qq.com, ${ }^{b} 757314739 @ q q$
}

Keywords: Car heating pad; Expert control; Intelligent control; Temperature regulation

\begin{abstract}
This paper aims at the disadvantages of traditional car heating pad without automatic starting function and temperature intelligent adjustment function, with single-chip computer control as the core, adopting multi-sensor fusion technology and expert control to realize the function of automatic switch of heating pad and automatic adjustment of temperature with the change of environment, then it has self-protection functions such as over flow, under pressure, over pressure, so as to improve the safety of driving and riding.
\end{abstract}

\section{Introduction}

As the quality of life improves, people have higher and higher requirements for comfort and intelligence of driving cars, in the cold winter, the internal temperature of the car is low, especially when the car first started, the air conditioning temperature will take a long time to reach the standard, the car heating pad have rapid heating, good control and adaptability, which can greatly improve the comfort of driver and passenger. At present, the temperature control of automobile heating pad is mostly realized through the construction of analog circuit or the control of single-chip computer, through the switch to control heating, through the knob to control the heating temperature, this paper design an intelligent temperature control system of heating pad based on expert control, using expert experience, control the heating pad temperature in real time according to the vehicle environment temperature and the driver's physical characteristics, at the same time, it has self-protection functions such as over-current, under-pressure, over-pressure and over-temperature, so as to improve the comfort and safety of drivers and passengers.

\section{Overall Scheme Design of the System}

Automobile battery is used as voltage starting output source, the $12 \mathrm{~V}$ voltage output from the voltage starting output source becomes the voltage value of $220 \mathrm{~V}$ after passing the inverter. this voltage is used as the final voltage source to heat the car heating pad, the heating pad is made of carbon fiber. When the car starts 10s, the MCU starts when a pressure sensor on a car seat detects a person's weight, it generates a switching signal, The heating pad enters the heating preparation work. If there is no open signal, the heating pad will not work. Intelligent control is selected for the heating process of heating pad: single-chip microcomputer AT89S51 is the core component. combined with temperature sensor, LCD display circuit and output control circuit, it can collect and control the working temperature of heating pad (heating and insulation).detect the heating pad voltage, current and temperature through the sensor. The basic block diagram is shown in Fig. 1. 


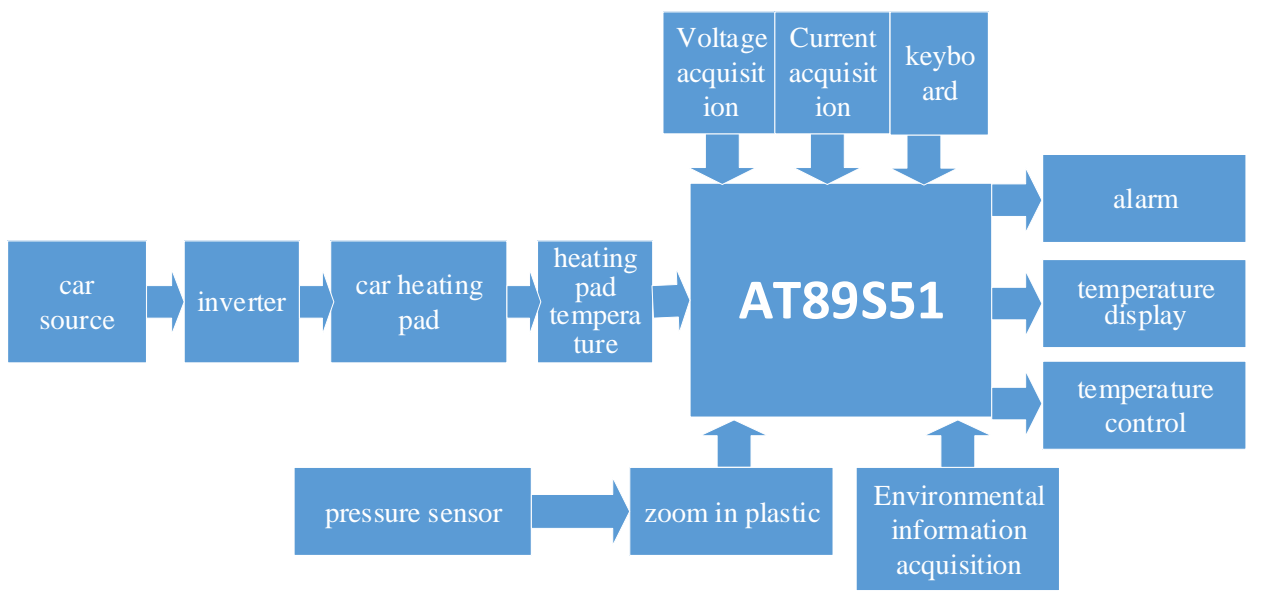

Figure 1. Finite Schematic diagram of double closed-loop speed regulating system

\section{System Hardware Design}

Power Output Unit. The power output unit includes the automobile power supply and inverter, automobile power is usually supplied by automobile battery or cigarette lighter, the inverter first converts the low voltage dc to the direct current of about $265 \mathrm{~V}$, the high voltage direct current is then converted to $220 \mathrm{~V}, 50 \mathrm{~Hz}$ ac. The power supply of the car takes full account of the external use environment, when overload or short circuit occurs, automatic protection will be shut down. The output voltage of the automobile power supply can be stabilized by its own feedback confirmation, the variation of no-load and rated voltage is less than $10 \mathrm{~V}$.

Data Acquisition Unit. The acquisition unit uses sensors to collect the temperature (ambient temperature, heating pad temperature) information and display the real-time temperature value. The acquisition of temperature information relies on the temperature sensor to sense the external temperature and transmit the temperature value to the single-chip microcomputer, real-time temperature display is to display the current temperature value of the heating pad. Temperature acquisition module adopted DS18B20digital temperature sensor.in this design, three DS18B2 were used to detect the environmental temperature, heating pad temperature and human face temperature in real time, the temperature information is processed by single chip microcomputer and sent to LCD1602 display screen.

Temperature Control Unit. The temperature control of heating pad is the core of this study, the heating pad of the car is a device to improve the comfort of the driver and passenger when the air conditioning in the car does not reach the comfort value. So whether the heating temperature is appropriate or not depends on how the body perceives the temperature, however, everyone has different constitution and driving habits, the thickness of clothes is also different (the thickness of clothes affects the perception of temperature),so it's hard to get a precise mathematical model of the temperature of the heating pad, conventional control theory cannot meet control requirements, at the same time, in order to improve the traditional heating pad temperature cannot be intelligently regulated, this system introduces expert control on the basis of traditional PID control mode, introducing expert knowledge and experience into the control system, through the sensor to measure the ambient temperature in the car, the heating pad is automatically adjusted to the corresponding temperature according to the difference of the inside environment and the difference of the dressing thickness of the body, the real-time temperature expert control system structure of automobile heating pad is shown in Fig. 2 below. 

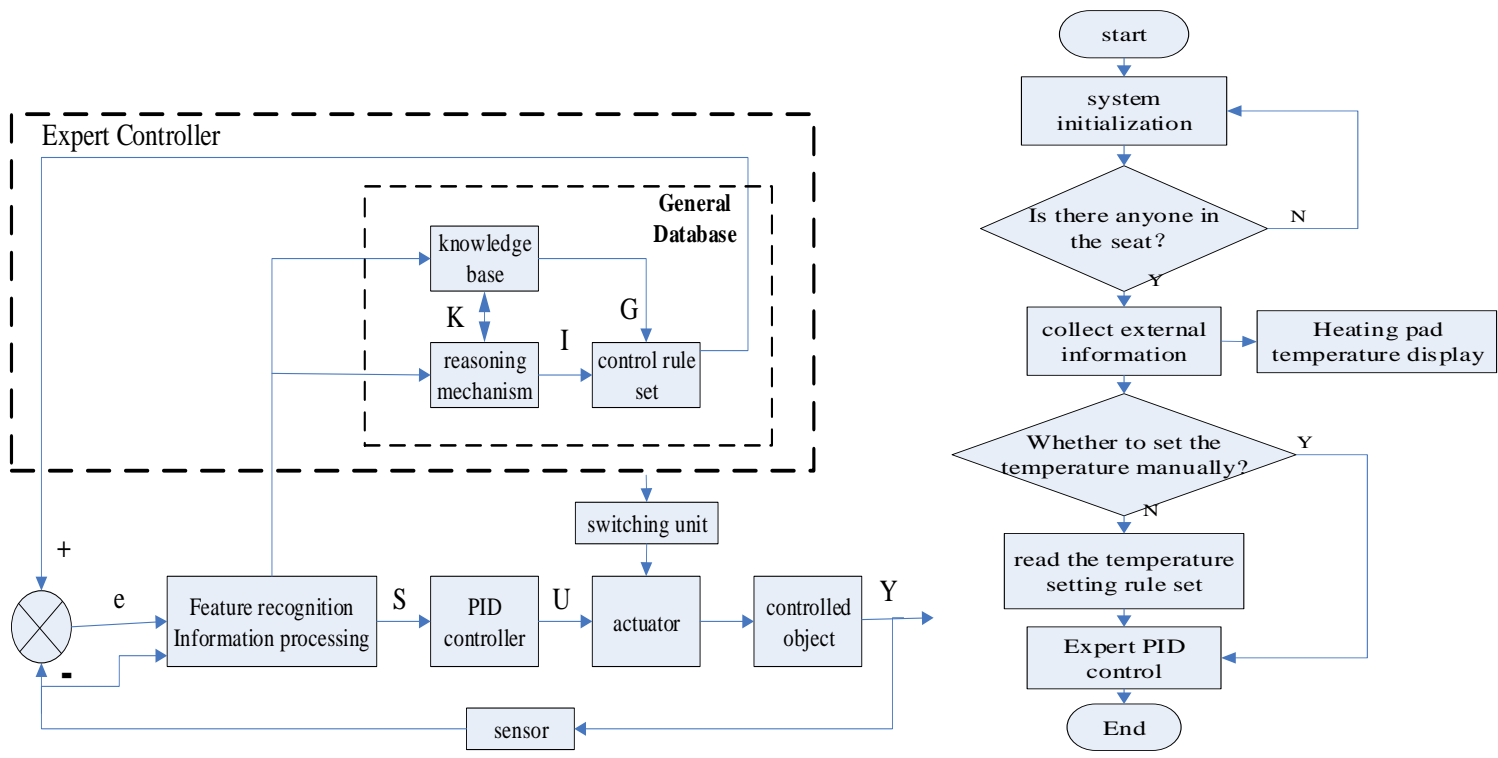

Figure 2. Finite expert controller structure diagram of automobile heating pad expert control system

Figure 3. Finite main program flow diagram

The basic control part of the real-time expert control system still adopts PID control, with data acquisition and closed-loop control functions. The external input information of the system enters the feature recognition and information management unit, and the information is processed and sent to the knowledge base and reasoning machine respectively. the knowledge base modifies the rules in the knowledge base according to the input information, the reasoning machine finds the corresponding control rules from the knowledge base, on the one hand, the set value given by experts is sent to the reference control input terminal, on the other hand, the expert will specify the action control executor.

1.Feature recognition and information processing

The function of feature recognition and information processing mechanism is to extract and process information and provide the basis for control decision and learning adaptation. It mainly includes extracting the characteristic information of dynamic process, identifying the characteristic state of the system, and making necessary processing of the characteristic information, feature recognition and information processing in addition to containing a heating pad and environment inside the car and the status of the parameters such as data human body, also includes error e in temperature control process, error change e 'and related variables and information in temperature control process.

2.Knowledge base

Knowledge base is the repository of expert knowledge, experience, theoretical knowledge and common sense. It is a very important part of expert system, the establishment of knowledge base is a process of continuous modification, expansion and improvement. knowledge expression in knowledge base adopts "IF(the premise is true) THEN ( conclusion the established)"generation knowledge expression, which is convenient for computer implementation and expert system development.

The temperature setting rule set is as follows:

IF T $<13^{\circ} \mathrm{C} \quad$ THEN $_{2}=70^{\circ} \mathrm{C}$

IF $13^{\circ} \mathrm{C}<\mathrm{T}<18^{\circ} \mathrm{C}$ and $\mathrm{T}_{1}<36^{\circ} \mathrm{C}$ THEN $\mathrm{T}_{2}=70^{\circ} \mathrm{C}$

IF $13^{\circ} \mathrm{C}<\mathrm{T}<18^{\circ} \mathrm{C}$ and $\mathrm{T}_{1}>36^{\circ} \mathrm{C}$ THEN $\mathrm{T}_{2}=60^{\circ} \mathrm{C}$

IF $18^{\circ} \mathrm{C}<\mathrm{T}<22^{\circ} \mathrm{C}$ and $\mathrm{T}_{1}<36^{\circ} \mathrm{C}$ THEN $\mathrm{T}_{2}=60^{\circ} \mathrm{C}$

IF $18^{\circ} \mathrm{C}<\mathrm{T}<22^{\circ} \mathrm{C}$ and $\mathrm{T}_{1}>36^{\circ} \mathrm{C}$ and $\mathrm{H}=$ "no sweating" $\mathrm{THEN} \mathrm{T}_{2}=50^{\circ} \mathrm{C}$

IF $18^{\circ} \mathrm{C}<\mathrm{T}<22^{\circ} \mathrm{C}$ and $\mathrm{T}_{1}>36^{\circ} \mathrm{C}$ and $\mathrm{H}=$ "sweating” $\mathrm{THEN} \mathrm{T}_{2}=40^{\circ} \mathrm{C}$

IF $\mathrm{T}>22^{\circ} \mathrm{C} \quad \mathrm{T}_{2}=0^{\circ} \mathrm{C}$ (stop heating)

Among them $\mathrm{T}$ is in-car ambient temperature, $\mathrm{T}_{1}$ is human facial skin temperature, $\mathrm{T}_{2}$ is heating 
pad preset temperature, $\mathrm{H}$ is human skin moisture.

Temperature control rule set:

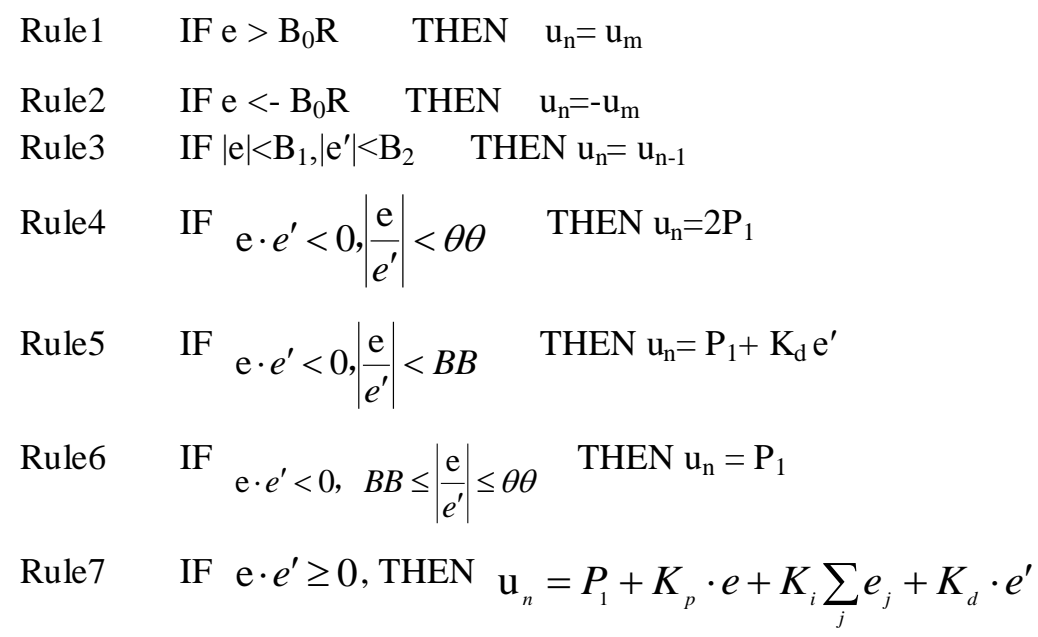

Among them $u_{n}$ and $u_{n-1}$ is the expert controller outputs the values in steps $n$ and $n-1 ; u_{m}$ is maximum output hold value; $\mathrm{P}_{1}$ is output hold before $R_{0} \cdot \sum_{j} \mathrm{e}_{j} ; \mathrm{R}_{0}$ is the weight coefficient (can be adjusted and corrected online); $\mathrm{B}_{1}$ is the allowable error; $\mathrm{B}_{2}$ is the allowable value of error change; $\mathrm{B}_{0}$ and $\theta \theta$ and $\mathrm{BB}$ is Experience parameters, it can be adjusted and corrected according to expert knowledge and experience.

The above 7 rules divide the dynamic response process of control system into 7 different states, different control rules are adopted in different states to realize the intelligence of PID control.

Because the control rule set has only 7 rules, its reasoning mechanism becomes very simple, so we can use forward reasoning mechanism.

3. Control process

According to the above rules, the control process is as follows:

(1). When the tracking error is large, the control function is strengthened to realize fast tracking adjustment (Rule1 and Rule2).

(2). When the error and its rate of change are within the allowable range, the output control function remains unchanged (Rule3).

(3). When the error is opposite to the symbol of its rate of change, and the error rate is relatively small, the original control is strengthened (Rule4).

(4).When the error is opposite to the symbol of its rate of change and the error rate is relatively large, the "differential" control is added (Rule5).

(5). When the symbol of error and rate of change is opposite, and the range of error and rate of change are small, the original control is maintained (Rule6).

6). When the error and the rate of change symbol are the same, the proportional - integral differential control is added to enhance the control effect (Rule7).

In the control process, the computer can easily identify the symbol and size of the error and its rate of change, so as to grasp the behavior characteristics of the dynamic process of the system, through intuitive reasoning, online determination or transformation of control strategies, flexible PID control is realized, which fully simulates human intelligent control and reflects the advantages of human-like intelligence.

Software design of the system. Overall design idea: the first is system initialization, the pressure sensor detects whether the seat is occupied and determines whether information is collected, then temperature sensors measure ambient temperature, pad temperature, facial temperature and humidity, the temperature value of the heating pad is read in the knowledge base, and then the temperature value of the heating pad is sent to the display screen after comparison calculation between the temperature value of the heating pad and the setting temperature value. The temperature of the heating pad can also be set by pressing the button, single-chip computer 
determines whether the external button is pressed down to set the temperature, then it is compared with the actual measured temperature, according to the comparison value to control the calorific value of the heating plate. the main flow diagram is shown in Fig. 3.

\section{Summary}

This paper takes single-chip microcomputer control as the core and adopts multi-sensor fusion technology and expert control technology, it makes up for the fact that the traditional car heating seat does not have automatic start function and temperature intelligent adjustment function, by heating the car pad hardware and software design, the intelligent temperature adjustment of car heating pad is realized, however, the system is imperfect. For example, the self-learning function of expert system needs to be developed and improved.

\section{References}

[1] Lili Li. design and research of car temperature regulating seat[D]. beijing:North China electric power university,2013.

[2] Bin Huang, Xinxing Luo. The utility model relates to a car seat cushion which can be cooled and heated[P]. china patent:200920243703.1, 2011-3-6.

[3] Pengyong Dong,Panxin Yuan,Qingli Niu. design of intelligent temperature detection system based on MSP430[J]. microprocessor,2010(6):75-78.

[4] Changwei Qu, Xingfu Zhu,Haiyang Jiang. research on design and application of PID temperature control system[J]. digital technology and applications.2017(02).

[5]Peng L, Fang W. Heterogeneity of Inferring Reputation of Cooperative Behaviors for the Prisoners' Dilemma Game [J]. Physica A: Statistical Mechanics and its Applications, 2015, 433: 367-378. 